\title{
Shifting from control to elimination: analysis of malaria epidemiological characteristics in Tengchong County around China-Myanmar border, 2005-2014
}

Shengguo Li², Shouqin Yin'², Jiazhi Wang ${ }^{2}$, Xishang $\mathrm{Li}^{2}$ and Jun Feng ${ }^{1 *}$

\begin{abstract}
Background: Tengchong County experienced a decreasing malaria prevalence period in 2005-2014 but the factors contributing to the trend are unclear. Herein, the malaria epidemiological data in years of 2005-2014 were collected and analysed, in order to provide evidence for subsequent effective strategic planning of malaria elimination that may be referenced by other counties with the similar elimination programmes along the China-Myanmar border.

Methods: A retrospective study was conducted to explore malaria-endemic characteristics in years 2005-2014 in Tengchong County. All individual cases from a web-based reporting system were reviewed and analysed. Local infections and imported cases were obtained from an annual reporting system.

Results: In total, 8321 confirmed malaria cases were recorded in this period, and $91.5 \%$ of them were reported during 2005-2010. Plasmodium vivax was the major species ( $n=5867,70.5 \%$ ). Most cases (92.9 \%) were found in males, mainly in the age group 30-34 years. Only five deaths resulting from Plasmodium falciparum were reported, of which three occurred in 2005. The cases were mainly reported in the townships of Wuhe (18.5\%), Mangbang (12.8\%) and Gudong (9.3\%). In addition, 147 local malaria (1.8\%) and 8174 imported malaria (98.2 \%) were observed during 2005-2014. However, the proportion of imported malaria was more than $95 \%$ all the time and no local transmission has been observed since 2013. Moreover, Myanmar was the main imported source, with 716 cases $(94.6 \%, 716 / 757)$ from Myanmar in 2011-2014.

Conclusions: Tengchong County has made achievements in controlling malaria, with incidence at historically its lowest level. However, imported malaria has increased and poses a great threat to malaria elimination. To achieve the elimination goal and prevent the re-introduction of malaria, surveillance systems need to be well planned and managed to ensure timely case detection and prompt response targeted to the mobile and migrate population at elimination stage.
\end{abstract}

Keywords: Malaria, Epidemiology, Tengchong County, Elimination, China-Myanmar border

\footnotetext{
*Correspondence: fjphilip1983@hotmail.com

1 National Institute of Parasitic Diseases, Chinese Center for Disease

Control and Prevention; Key Laboratory of Parasite and Vector Biology,

Ministry of Health; WHO Collaborating Centre for Tropical Diseases,

National Center for International Research on Tropical Diseases,

200025 Shanghai, China

Full list of author information is available at the end of the article
} 


\section{Background}

Malaria is caused by one or more of the five species of Plasmodium via the bite of infected female Anopheles mosquitoes. An estimated 214 million confirmed cases were reported from 75 countries and territories in 2014, with 438,000 deaths [1]. China has made obvious achievements in controlling locally transmitted malaria over the past decades and incidence was down to historically low levels in 2009 [2, 3]. China initiated the National Malaria Elimination Programme (NMEP) in 2010, which aimed to eliminate indigenous malaria, except for border areas, by 2015 , and to completely eliminate nationwide by 2020 [4].

Despite this, malaria along the border regions is still a great challenge to the elimination process. For example, although there was a $99.1 \%$ decrease in local cases reported from $2005(\mathrm{n}=5119)$ to $2014(\mathrm{n}=47)$ in 18 counties along the China-Myanmar border, this still accounts for $82.5 \%$ of total local cases throughout the whole country [5]. Tengchong County is one of the 18 border counties with a total population of $6,681,000$ and a border line of $148 \mathrm{~km}$, located in the southwest of Yunnan Province. It has 18 townships of which three are bounded by Kachin State Special Region-1, Myanmar (Fig. 1). Tengchong County was an endemic area, prone to large outbreaks and classified a malaria-unstable region [4]. Frequent migration, wide distribution of Anopheles mosquitoes, and poor treatment contributed to the high incidence and recurrence in Tengchong County [6]. For instance, 2709 cases occurred in 2005, a $34.6 \%$ increase on 2004, mainly due to an arrival of migrant workers from Myanmar during December 2004April 2005 [7].

Another risk is that malaria in the Sino-Myanmar border areas has not been effectively controlled [8]. Wang conducted a survey in four special regions of northern Myanmar, near China and showed that the average prevalence of malaria infection was $13.6 \%$ [9]. In Kachin State Special Region-1, neighbouring Tengchong County, the main source of local income is grain cultivation, coupled with income from scattered rubber and sugar cane cultivation. Local people live in extreme poverty and

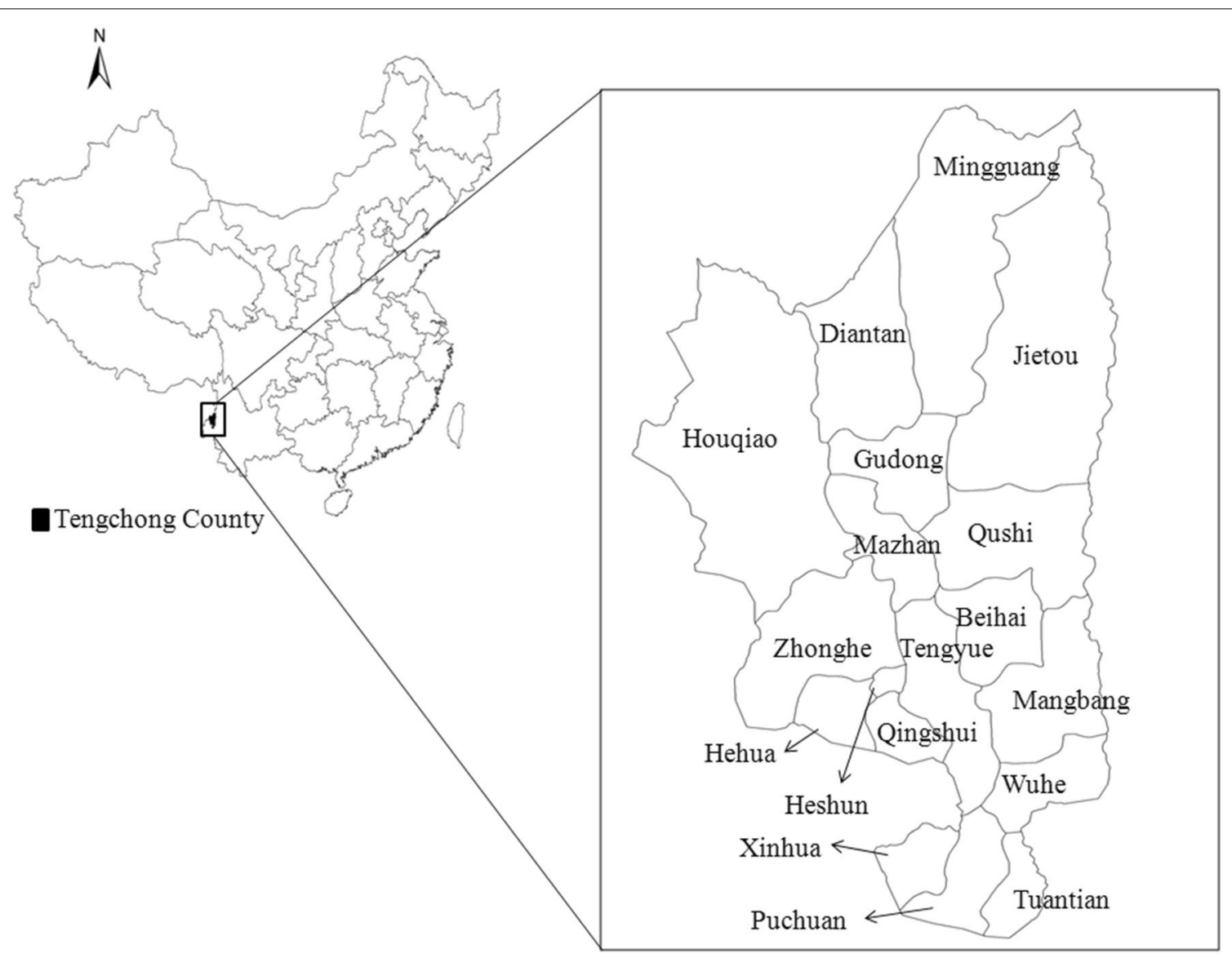

Fig. 1 Location of Tengchong County in China. The names of 18 townships are labelled on the right 
education levels are generally low. In these communities, socio-economic under-development, an unstable political situation, relatively weak healthcare infrastructure, and difficult access to healthcare services all provide challenges to implementing and maintaining effective malaria prevention and control. The long China-Myanmar border, which has no natural barriers, is subject to frequent cross-border migration, making it difficult to manage a migrating population through appropriate channels.

In this finding, the malaria epidemiology in Tengchong County was characterized during 2005-2014, an important transition period from control to elimination phase, in order to provide evidence-based proof to support adjustment of appropriate strategies and interventions towards malaria elimination, which could be referenced by other counties along the China-Myanmar border.

\section{Methods}

\section{Data collection}

A retrospective study was conducted to explore malariaendemic characteristics during 2005-2014 in Tengchong County. All individual cases from a web-based reporting system (WBRS) were carefully reviewed and analysed. The data were selected by onset of date, reporting area and final confirmation. The WBRS parameters consisted of species composition, geographical distribution, gender and age distribution of cases, and number of deaths. Clinically diagnosed cases were defined as a patient with history of travel to an malaria-endemic area and with malaria-like symptoms but no parasites detected by blood examination. Laboratory-confirmed cases were defined as using any of the laboratory tests, including polymerase chain reaction (PCR), rapid diagnostic tests (RDT) and microscopy examination. Both clinical malaria and confirmed malaria cases were included in the present study. For the total number of cases of local infections and imported malaria cases, data were obtained from another system, an annual reporting system. In China, any cases defined as imported malaria must meet all of the following criteria: (1) by giving a diagnosis of malaria; (2) with a travel history to malaria-endemic areas outside China during malaria transmission season; and, (3) the onset time of the patient being less than one month after returning to China during the local transmission season. This definition is based on the reasonable incubation period for all Plasmodium species reported in China [10].

\section{Data analysis}

A descriptive analysis was processed using Microsoft Excel and SAS software (SAS Institute Inc, Version 9.2, Cary, NC, USA). Distribution maps of imported malaria in 2005, 2010 and 2014 were created by ArcGIS 10.1 (Environmental Systems Research Institute, Inc, Redlands, CA, USA). Annual parasitic incidence $(\mathrm{API})=$ total confirmed cases in a year $\times 1000 /$ total population.

\section{Results}

A total of 8321 malaria cases were recorded by WBRS during 2005-2014, among whom 7615, accounting for $91.5 \%$ of all cases, were reported from 2005 to 2010 [5, 11-18]. During this period, reported malaria cases reached a peak in $2006(\mathrm{n}=2206)$ with the highest incidence $(35.4 / 10,000)$ (Fig. 2a). Unlike Yunnan Province and the whole country, the proportion of imported cases remained at high degree all the time in Tengchong County, 2005-2014 (Fig. 2b).

Cases in Tengchong County declined annually from 2010, when the malaria elimination programme was launched. There was a $76.6 \%$ decrease noted from 2010
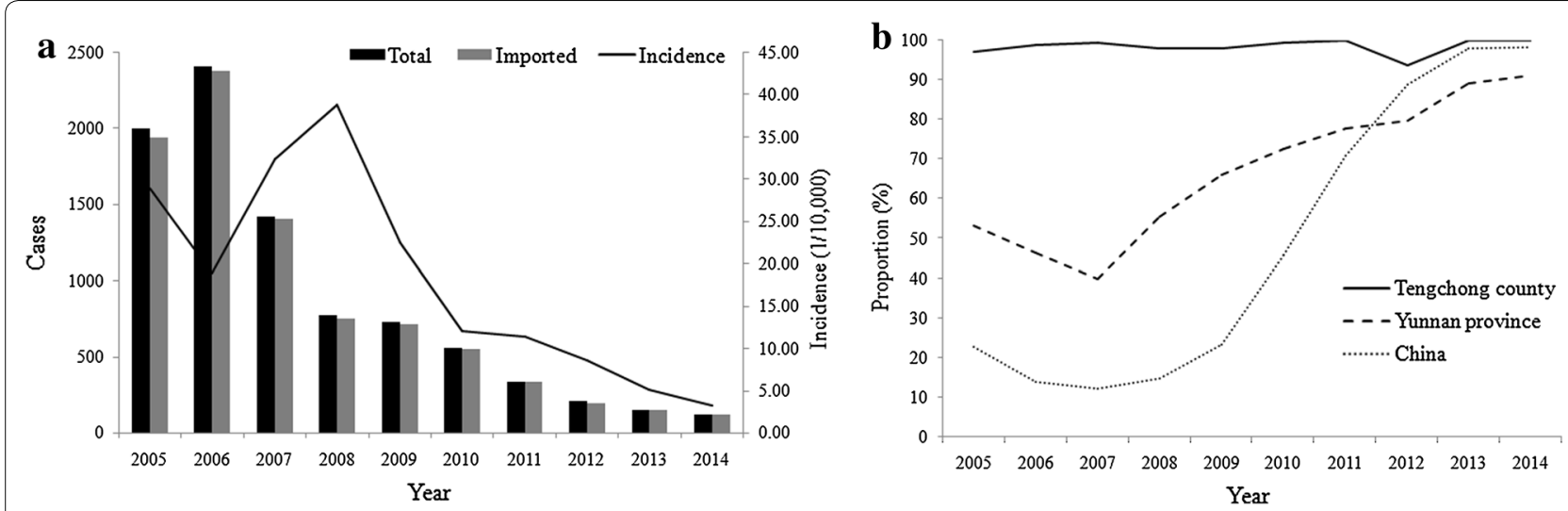

Fig. 2 Malaria prevalence in Tengchong County, 2005-2014. a The columns show the change trend of total cases (black) and those imported from other countries (grey), respectively. The black line indicates malaria incidence during 2005-2014. b The trend of proportion of imported cases in Tengchong County, Yunnan Province and China 
( $\mathrm{n}=556)$ to $2014(\mathrm{n}=130)$, and no local transmission has been observed since 2013. Malaria cases occurred in males $92.9 \%(\mathrm{n}=7733)$ and $7.1 \%$ in females $(\mathrm{n}=588)$ with similar distribution for all age groups. Most malaria cases were observed in the age group 30-34 years, $94.1 \%$ in males and $5.9 \%$ in females. Cases were mainly reported April-October and December-February, when migrate workers returned for farm work and to enjoy the Spring Festival, respectively (Fig. 3).

In addition, WBRS reported 931 clinically diagnosed (11.2\%) and 7390 laboratory-confirmed cases (88.8 \%) from 2005 to 2014, respectively. The proportion of clinically diagnosed cases had decreased since 2006, and since 2013 there were no clinically diagnosed cases reported in Tengchong County. From 2005 to 2014, WBRS reported five malaria deaths $(0.06 \%, 5 / 8321)$, all Plasmodium falciparum infections; Jietou, Mazhan, Hehua, Qingshui, and Wuhe reported one death each. The highest number of deaths occurred in $2005(n=3)$.

\section{Plasmodium species}

In total, 5867 Plasmodium vivax and 1783 P. falciparum cases were recorded in Tengchong County, 2005-2014
(Fig. 4). The proportion of $P$. falciparum gradually reduced from $11.5 \%$ in 2014 , down by $59.4 \%$ compared with 2005. All the townships had a higher proportion of $P$. vivax (the mean $P$. vivax to $P$. falciparum $=3.3-1$ ). The highest ratio of $P$. vivax to $P$. falciparum was observed in Puchuan Township with 6.4 and lowest ratio occurred in Heshun Township with 2.3.

\section{Regional distribution}

Malaria cases were mainly distributed in three townships: Wuhe ( $\mathrm{n}=1542,18.5 \%)$, Mangbang ( $\mathrm{n}=1066$, $12.8 \%$ ) and Gudong ( $\mathrm{n}=774,9.3 \%)$. The API for each township decreased gradually. For instance, the highest API was observed in Xinhua Township with 17.7 in 2005, while in 2014 the highest API occurred in Wuhe Township with 0.59. Additionally, the south part was the severe region as the API for this area was almost the highest in Tengchong County from 2005 to 2014 (Fig. 5).

\section{Source of infection}

Data from annual reporting system of 2005-2014 indicated 147 local malaria (1.8\%) and 8174 imported malaria (98.2 \%) cases. During this period, local transmission decreased by $77.6 \%$ from $2005(n=58)$ to $6.3 \%$

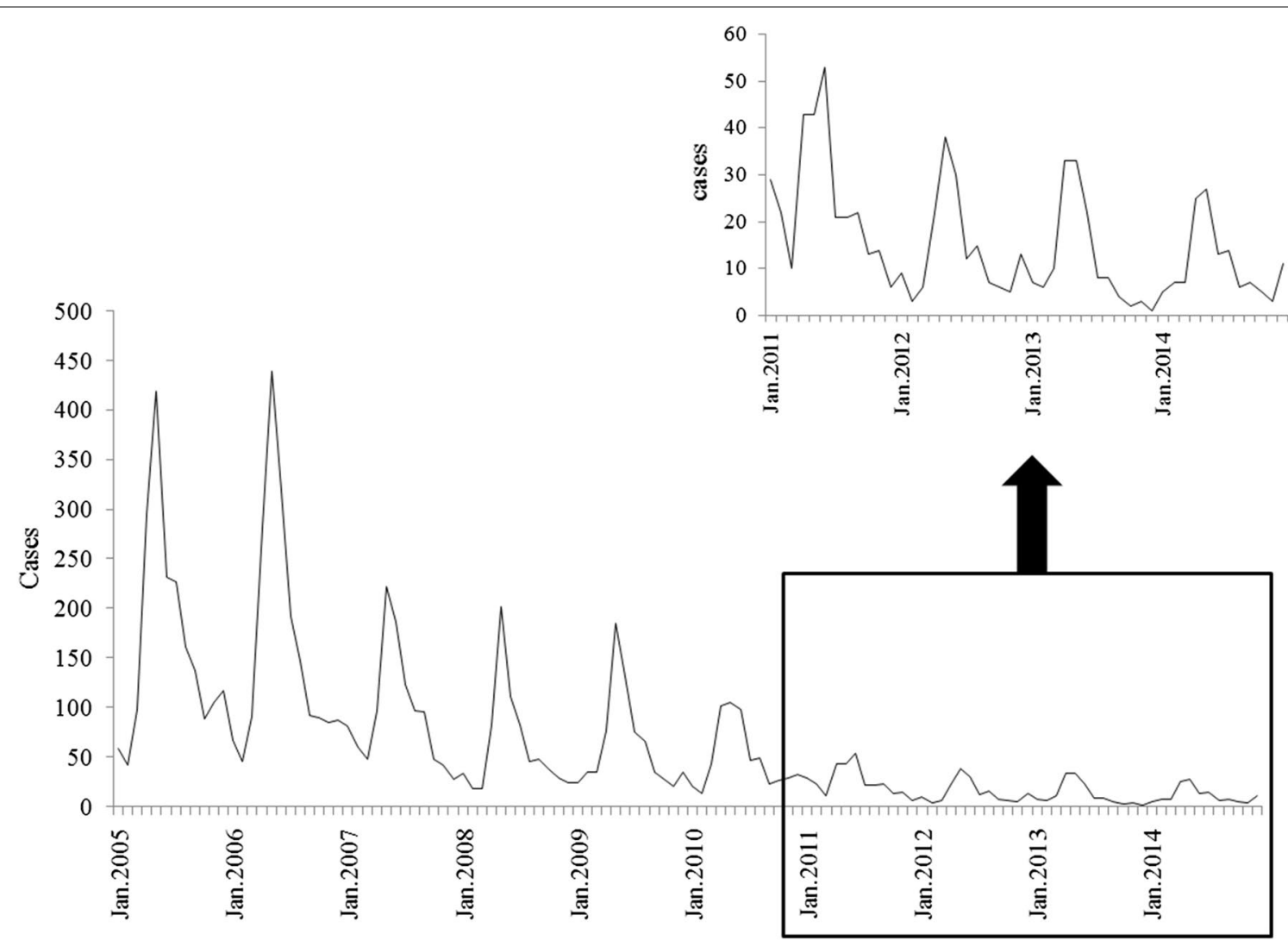

Fig. 3 Monthly distributions in Tengchong County, 2005-2014 


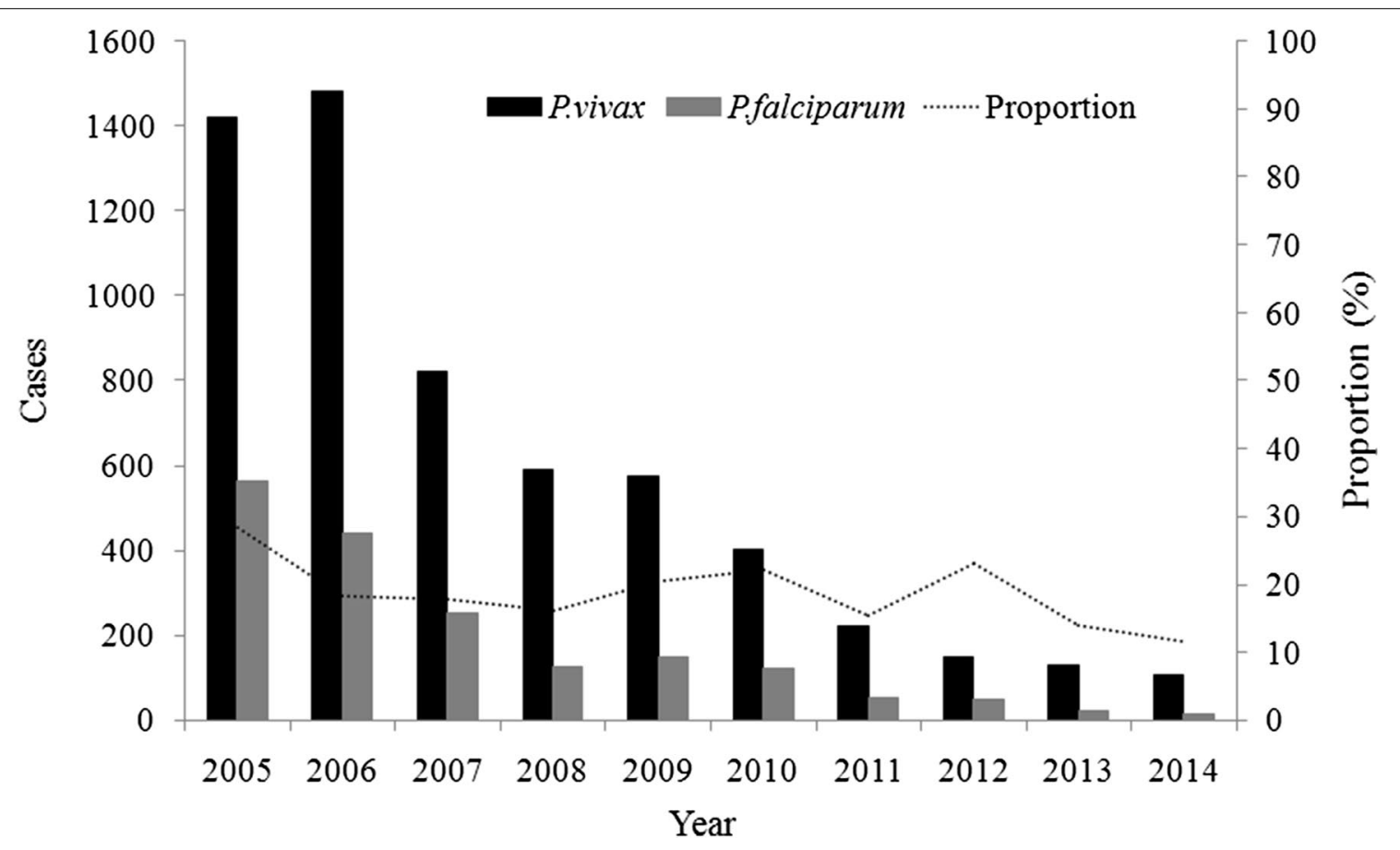

Fig. 4 Trend of Plasmodium vivax and Plasmodium falciparum, Tengchong County, 2005-2014. The lines of different columns show the change trend of P. vivax (black) and P. falciparum (grey). The dash line represents the proportion of P. falciparum in the total cases

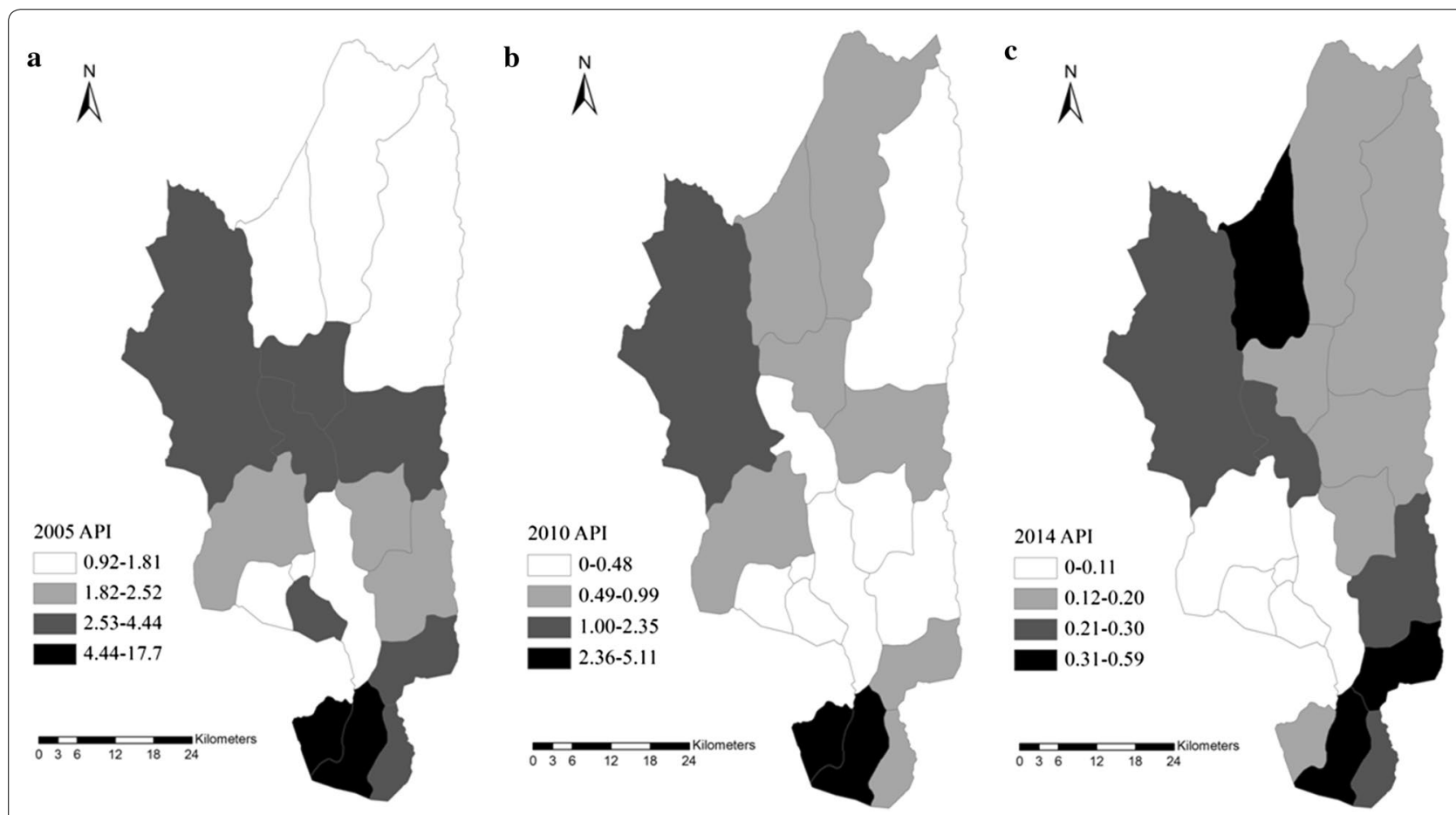

Fig. 5 Annual parasite index distribution in Tengchong County, in 2005 (a), 2010 (b) and 2014 (c). Map created by ArcGIS 10.1. The different coloured squares indicate reported case number ranges 
in $2012(\mathrm{n}=13)$, and since 2013 no local cases have occurred. Local cases were mainly reported in the townships of Qushi $(\mathrm{n}=29,19.7 \%)$, Jiehe $(\mathrm{n}=18,12.2 \%)$ and Zhonghe ( $\mathrm{n}=12,8.2 \%)$. Local P. falciparum $(\mathrm{n}=49)$ occurred mainly in Qushi $(\mathrm{n}=17)$ and Jiehe townships $(\mathrm{n}=7)$. Interestingly, Puchuan reported one local Plasmodium malariae in 2009, confirmed both by microscopy and RDT.

Contrary to decreasing local transmission, the proportion of imported malaria has continuously increased from 2005 (97.1 \%) to 2014 (100.0 \%). Of the WBRSrecorded 757 imported malaria cases during 2011-2014 in Tengchong County, 716 cases $(94.6 \%)$ were returned from Myanmar. The cases imported from Myanmar observed a $46.4 \%$ decrease from $2011(\mathrm{n}=282)$ to 2014 $(\mathrm{n}=112)$. This was probably because the decrease in malaria cases also occurred in the Myanmar. The World Health Organization (WHO) reported that the number of cases reported in 2013 (333871) decreased by $30.2 \%$ when compared to $2012(\mathrm{n}=478084)$ in Myanmar [1, 19].

\section{Discussion}

Tengchong County is one of 18 border counties on the China-Myanmar border classified into Type II counties according to NMEP, with an emphasis on disposal of any possible malaria cases and active foci in order to interrupt local transmissions [4].

Tengchong County had experienced a declined period during 2005-2014. There are several factors that contribute to the decreasing trend in malaria prevalence in Tengchong County, 2005-2014: firstly, Tengchong County obtained the support of the Global Fund Rounds five and six, which provides a financial guarantee and offers help with malaria control. For example, the number of blood detections and insecticide-treated net (ITN) distribution was 25466 and 36093 in 2011, increasing by 105.3 and $409.9 \%$ compared with 2006 , respectively; secondly, China launched NMEP and more strict requirements on interventions, including epidemiological survey, vector control, data or sample collection and storing, etc., which facilitates intervention implementation to progress malaria elimination. In 2012, Tengchong County Center for Disease Control and Prevention (CDC) was chosen as the malaria elimination base co-operated by the National Institute of Parasitic Diseases (NIPD), this strengthens technical support and promotes the elimination process; thirdly, the establishment of joint control and prevention mechanism in 2010 facilitated information exchange and communication with Kachin State Special Region-1 of Myanmar and six information exchange meetings were held until 2011; finally, the Chinese Government prohibited illegal logging from Myanmar since 2006, which has largely decreased importation of malaria in patients returning from Myanmar [20].

Another characteristic in Tengchong County is the high proportion of imported cases all the time. Unlike the whole country, importation was more than $95 \%$ all the time in 2005-2014 except 2013, while the proportion of imported cases in Yunnan Province and the whole country gradually increased after 2007 [21]. This was because of the frequent movement of population going to Myanmar for trading, migration and travel. The high proportion of imported cases brings high risk to local facilities, as well as the wide distribution of Anopheles mosquitoes, which may cause secondary infection and outbreak epidemic [22]. Therefore, how to identify imported infections from neighbouring countries is crucial and has been a great challenge especially in border regions. WHO recommends the screening for malaria infection at border checkpoints not only to identify and treat malaria cases, but also to set up response measures to prevent re-introduction across the border. More active measures may be needed at border immigration checkpoints, to target longer-term migrant workers [23]. To solve this, Tengchong County has launched four border malaria posts and screens febrile patients for malaria by RDTs and microscopy. The posts also provide an outreach service to nearby villages for active detection using RDT and deliver Information, Education and Communication (IEC) materials.

Combined with importation, the emergence of resistance to artemisinin found on the Thai-Cambodian border has also become a great concern for malaria elimination in Tengchong County [24, 25]. Although artemisininbased combination therapy (ACT) is generally effective in treating $P$. falciparum in Tengchong County, there has been some indication of reduced sensitivity of parasites to artemisinin and its derivatives, and some mutation of genotype against $P$. falciparum observed along the ChinaMyanmar border [26, 27]. Rigorous clinical efficacy studies are still warranted in this region due to frequent usage of monotherapy over several decades.

To deal with imported cases, a surveillance system should be established to target mobile and migrant populations and ensure timely recognition, prompt response and effective management. Accurate RDT and PCR confirmation should be developed for efficient surveillance [28]. CDC staff should carry out screening work on high-risk populations of Chinese nationals returning to Tengchong County (in communities) and for Myanmar migrants coming to Tengchong County. What is also needed is for the existing cooperation mechanism to remain, such as joint prevention and control between China and Myanmar, and for the establishment 
of an information-sharing platform of surveillance and response. Additional steps can be taken to prevent malaria resurgence, including mosquito control measures in the areas with high receptivity and improved accessibility to qualified anti-malarials and compliance of treatment. If these strategies are undertaken it will greatly reduce the risk of malaria re-introduction, and speed up achievement of the goal of malaria elimination in Tengchong County.

\section{Conclusions}

Malaria prevalence in Tengchong County has significantly declined in the past decade. However, the proportion of imported malaria by frequent mobile and migration populations will pose a great threat to the elimination programme. Therefore, surveillance systems need to be carefully planned and well managed to ensure timely recognition and prompt response to imported cases as well as to remove any residual local cases. In addition, effective mechanism of multisectoral coordination and cooperation, joint prevention and control should be strengthened. The health authority should strengthen verification and trace investigation of any malaria cases, including indigenous and imported ones, which are required to ensure elimination of any potential reservoir and prevention of local transmission caused by imported pathogens.

\section{Authors' contributions}

SQY and XSZ conceived the study and undertook statistical analysis. SGL initiated the study and provided useful suggestions. JF made major contributions to drafting the manuscript. All authors contributed to the writing of the manuscript and approved the submitted version of the manuscript. All authors read and approved the final manuscript.

\section{Author details \\ ${ }^{1}$ National Institute of Parasitic Diseases, Chinese Center for Disease Control and Prevention; Key Laboratory of Parasite and Vector Biology, Ministry of Health; WHO Collaborating Centre for Tropical Diseases, National Center for International Research on Tropical Diseases, 200025 Shanghai, China. ${ }^{2}$ Tengchong County Centers for Disease Control and Prevention, No. 51 Guan- ghua village, Tiancheng district, 679100 Tengchong, Yunnan Province, China.}

\section{Acknowledgements}

We thank the Key Laboratory of Parasite and Vector Biology, MOH (Grant No. wsbktkt201403) for financial support and all staff of Tengchong County Centers for Disease Control and Prevention in China for assistance.

\section{Competing interests}

The authors declare that they have no competing interests.

Received: 7 July 2015 Accepted: 3 January 2016

Published online: 28 January 2016

\footnotetext{
References

1. WHO: World malaria report: 2015. Geneva: World Health Organization; 2015
}

2. Li YN, Wen L, Li CY, Zhang WY, Sun HL, Zou W, et al. Descriptive analysis of trends of malaria prevalence in China from 2006 to 2010. Chin J Pub Health. 2013;29:263-5.

3. Feng J, Xia ZG, Vong S, Yang WZ, Zhou SS, Xiao N. Preparedness for malaria resurgence in China: case study on imported cases in 2000-2012. Adv Parasitol. 2014;86:231-65.

4. Chinese Center for Disease Control and Prevention: National Malaria Elimination Programme. Beijing; 2010.

5. Zhou SS, Wang Y, Tang LH. Malaria situation in the People's Republic of China in 2005. Chin J Parasitol Parasit Dis. 2006;24:401-3.

6. Yin SQ, Li XS, Kang XH, Li SQ, Wang XZ, Sun XD, et al. Preliminary inves tigation on malaria vectors Anopheline species in Tengchong County, Yunnan Province. Int J Med Parasit Dis. 2013:40:37-9.

7. Sun XDLJ, Yang YH, Duan YZ. Survey of recurrence of malaria infection in Tengchong County, Yunnan Province. Chin Trop Med. 2006;6:2155-6.

8. Richards AK, Banek K, Mullany LC, Lee Cl, Smith L, Oo EK, et al. Cross-border malaria control for internally displaced persons: observational results from a pilot programme in eastern Burma/Myanmar. Trop Med Int Health. 2009;14:512-21.

9. Wang RB, Zhang J, Zhang QF. Malaria baseline survey in four special regions of northern Myanmar near China: a cross-sectional study. Malar J. 2014;13:302.

10. Feng J, Yan H, Feng XY, Zhang L, Li M, Xia ZG, et al. Imported malaria in China, 2012. Emerg Infect Dis. 2014;20:1778-80.

11. Zhou SS, Wang Y, Tang LH. Malaria situation in the People's Republic of China in 2006. Chin J Parasitol Parasit Dis. 2007;25:439-41.

12. Zhou SS, Wang Y, Fang W, Tang LH. Malaria situation in the People's Republic Of China in 2007. Chin J Parasitol Parasit Dis. 2008;26:401-3.

13. Zhou SS, Wang Y, Fang W, Tang LH. Malaria situation in the People's Republic of China in 2008. Chin J Parasitol Parasit Dis. 2009;27(457):455-6.

14. Zhou SS, Wang Y, Xia ZG. Malaria situation in the People's Republic Of China in 2009. Chin J Parasitol Parasit Dis. 2011;29:1-3.

15. Zhou SS, Wang Y, Li Y. Malaria situation in the People's Republic of China in 2010. Chin J Parasitol Parasit Dis. 2011;29:401-3.

16. Xia ZG, Yang MN, Zhou SS. Malaria situation in the People's Republic of China in 2011. Chin J Parasitol Parasit Dis. 2012;30:419-22.

17. Xia ZG, Feng J, Zhou SS. Malaria situation in the People's Republic of China in 2012. Chin J Parasitol Parasit Dis. 2013:31:413-8.

18. Zhang L, Feng J, Xia ZG. Malaria situation in the People's Republic of China in 2013. Chin J Parasitol Parasit Dis. 2014;32:407-13.

19. WHO: World Malaria Report: 2013. Geneva: World Health Organization; 2013

20. Duan YZ, Li SG, Kang XH, Yin SQ, Ma ZX. Analysis of the prevalence of malaria in Tengchong County, Yunnan Province from 2005 to 2010. J Pathol Biol. 2011;6:533-5.

21. Feng J, Xiao H, Xia Z, Zhang L, Xiao N. Analysis of Malaria Epidemiological Characteristics in the People's Republic of China, 2004-2013. Am J Trop Med Hyg. 2015;93:293-9.

22. Duan YZ, Li SG, Kang XH, Yin SQ, Sun XD. A point-like outbreak caused by secondary transmission from an imported malaria vivax case. Int J Med Parasit Dis. 2013;40:57-9.

23. Yangzom T, Gueye CS, Namgay R, Galappaththy GN, Thimasarn K, Gosling $R$, et al. Malaria control in Bhutan: case study of a country embarking on elimination. Malar J. 2012;11:9.

24. Dondorp AM, Nosten F, Yi P, Das D, Phyo AP, Tarning J, et al. Artemisinin resistance in Plasmodium falciparum malaria. N Engl J Med. 2009;361:455-67.

25. Ashley EA, Dhorda M, Fairhurst RM, Amaratunga C, Lim P, Suon S, et al. Spread of artemisinin resistance in Plasmodium falciparum malaria. N Engl J Med. 2014;371:411-23.

26. Yang H, Liu D, Yang Y, Fan B, Yang P, Li X, et al. Changes in susceptibility of Plasmodium falciparum to artesunate in vitro in Yunnan Province, China. Trans R Soc Trop Med Hyg. 2003;97:226-8.

27. Feng J, Zhou D, Lin Y, Xiao H, Yan H, Xia Z. Amplification of pfmdr1, pfcrt, pvmdr1, and K13 propeller polymorphisms associated with Plasmodium falciparum and Plasmodium vivax isolates from the China-Myanmar border. Antimicrob Agents Chemother. 2015;59:2554-9.

28. Zhou X, Huang JL, Njuabe MT, Li SG, Chen JH, Zhou XN. A molecular survey of febrile cases in malaria-endemic areas along China-Myanmar border in Yunnan province, People's Republic of China. Parasite. 2014;21:27. 